\title{
A case control study of HIV seroconversion in gay men, 1988-1993: what are the current risk factors?
}

\author{
D I Williams, J M Stephenson, G J Hart, A Copas, A M Johnson, I G Williams
}

\begin{abstract}
Objective: To investigate current risk factors for HIV seroconversion among homo/bisexual men.

Design: Matched case control study in a large STD clinic in central London. Data on risk factors were obtained by case note review.

Methods: Fifty-six cases who had at least one negative HIV test followed by a positive test between June 1988 and July 1993, and two homo/bisexual controls (having two or more negative HIV tests) matched to each case on age, total number of HIV tests and test interval period were identified. Univariate and multivariate odds ratio were calculated for acute STD, ano-genital intercourse, condom use and HIV status of sexual partners.

Results: Adjusted odds ratios (95\% confidence intervals) for HIV seroconversion were $4 \cdot 1$ $(1 \cdot 3-13 \cdot 3)$ for having an acute interval STD and 4.6 (1.4-15.4) for having a known HIV infected sexual partner. Compared with men who always used condoms, odds ratios for men who sometimes or never used condoms were $7 \cdot 9(2 \cdot 2-28 \cdot 9)$ and $16 \cdot 2(3 \cdot 0-86 \cdot 0)$ respectively. Unprotected ano-genital intercourse was commonly reported by both cases and controls, and reported condom use was no greater with a known HIV infected partner than with a partner of unknown HIV status.

Conclusion: HIV seroconversion among homo/bisexual men attending STD clinics is strongly related to having an acute STD, a known HIV infected partner and not using condoms. Although consistent use of condoms is highly protective, knowing that a partner is HIV infected does not ensure condom use between serodiscordant men. More effective, well-evaluated interventions are needed to reduce sexual risk-taking in this population.

(Genitourin Med 1996;72:193-196)
\end{abstract}

Keywords: HIV; seroconversion; homosexual men

\section{Introduction}

It is well established from studies of gay and bisexual men in the USA, Europe and Australia that there was a major move towards the adoption of "safer sex" in the mid-late eighties. ${ }^{12}$ However, whilst these trends may apply on a population basis, unsafe sex remains a significant problem. Early in the 1990 s there was an increase in the incidence of rectal gonorrhoea (taken as a proxy marker of unsafe sexual behaviour) in gay men in London, ${ }^{3}$ a relatively high prevalence of HIV-1 infections in young gay men, ${ }^{4}$ and a continuing high prevalence of infection in gay and bisexual men in England. ${ }^{5}$ In London the estimated incidence of seroconversion in 1759 men who had repeat tests between 1988 and 1994 was 3.8 per 100 person years, with a higher incidence in younger gay men. ${ }^{6} \mathrm{~A}$ seroprevalence of $6-8 \%$ in young homosexual men under 25 years attending GUM clinics suggests that new infections are occurring early in their sexual histories. ${ }^{7}$

It is also evident ${ }^{8}$ that the epidemic of AIDS in the UK is set to continue, rising to an estimated annual incidence of 1505 AIDS cases by 1997 in men for whom transmission occurred through homosexual intercourse. This figure is based on estimates of the number of men who are currently infected with HIV who will go on to develop an AIDS defining disease. The back projections for HIV inci- dence are approximately 500 per annum between 1986 and $1991 .{ }^{8}$ There is a pressing need to understand better the present dynamics of the HIV epidemic, and in particular current risk factors for infection.

Longitudinal and cross-sectional studies have identified risk factors associated with transmission of HIV, including unprotected ano-genital intercourse, number of sexual partners, a previous history of sexually transmitted diseases (syphilis, gonorrhoea) and possibly the stage of HIV disease in the index case. ${ }^{9-11}$ As HIV has become more prevalent in populations of gay men, the risk of HIV infection from unprotected intercourse with a new partner has increased.

Despite widespread education campaigns homosexual men are continuing to become infected. To establish current risk factors for the continuing transmission of HIV infection we undertook a case control study of homosexual men attending a central London STD clinic for repeat HIV antibody testing during the 5 year period 1988-1993 comparing those who seroconverted with those who did not.

\section{Patients and methods}

All patients who had attended the clinic for at least two HIV antibody tests between June 1988 and July 1993 were identified from laboratory records of HIV antibody test results and 
Table 1 Reported sexually transmitted diseases and ano-genital intercourse in cases and controls

\begin{tabular}{lll}
\hline $1 . S T D$ & $n(\%)$ of cases & $n(\%)$ of controls \\
\hline 1 or more STD & $27(48)$ & $25(24)$ \\
2 or more STDs & $11(20)$ & $6(6)$ \\
1 or more episode of: & $14(25)$ & $9(9)$ \\
GC (any site) & $7(13)$ & $5(5)$ \\
GC (rectal) & $11(20)$ & $11(11)$ \\
NSU & $4(7)$ & $1(1)$ \\
HSV (primary) & $26(46)$ & $50(38)$ \\
or more STD before interval & $n(\%)$ of cases & $n(\%)$ of controls \\
2. Ano-genital intercourse & $4(7)$ & $9(9)$ \\
None & $15(27)$ & $3(3)$ \\
Receptive only & $3(5)$ & $17(17)$ \\
Insertive only & $34(61)$ & $75(72)$ \\
Both &
\end{tabular}

GC: gonorrhoea; NSU: non-specific urethritis; HSV: herpes simplex virus.

an in-house data base recording attendance and diagnosis. Fifty-six patients, all homosexual men with no other risk factor, were found to have seroconverted for HIV.

All patients undergoing repeat HIV testing at the clinic and remaining negative were identified from the same sources. A control group of 104 homosexual men were selected and matched for age, total number of HIV antibody tests and comparable interval period. Each seroconverter was matched with two controls, except in 8 cases where only one suitable control each could be identified.

The mean age of cases was $29 \cdot 7$ years (SD 5.5, range 18-24), and of controls was 31.9 years (SD 6.0, range 22-46). The median test interval was 10.5 months (range 1-42) for cases and 10 months (range 1-39) for controls. Fifty-eight (56\%) of the controls and 29 $(52 \%)$ of the cases had had two tests, and the range was 2-8 for controls and 2-7 for cases.

We aimed to select controls aged within 6 years of the age of the case, but this was sometimes difficult, and 18 controls were outside this range. Cases were significantly younger than the mean age of their control(s) (mean difference 2.4 years, $\mathrm{p}<0.0005$, paired $t$ test). Matching on interval and number of tests was successful, all but three controls matched at six months or less from the interval of the case, and all but two controls matched exactly or \pm one to the number of tests of the case. For the

Table 2 Univariate analysis of factors associated with seroconversion

\begin{tabular}{|c|c|c|c|}
\hline Factor & & Odds ratio & $\begin{array}{l}\text { 95\% confidence } \\
\text { interval }\end{array}$ \\
\hline STD during interval & $\begin{array}{l}\text { None } \\
1 \text { or more }\end{array}$ & $\begin{array}{l}1 \\
3 \cdot 5\end{array}$ & $1 \cdot 6-7 \cdot 6$ \\
\hline Previous STD & $\begin{array}{l}\text { None } \\
1 \text { or more }\end{array}$ & $\begin{array}{l}1 \\
0.96\end{array}$ & $0 \cdot 49-1 \cdot 90$ \\
\hline Condom use & $\begin{array}{l}\text { Always } \\
\text { Sometimes } \\
\text { Never }\end{array}$ & $\begin{array}{c}1 \\
4.5 \\
10 \cdot 9\end{array}$ & $\begin{array}{l}1 \cdot 9-10 \cdot 2 \\
3 \cdot 3-36 \cdot 0\end{array}$ \\
\hline Ano-genital intercourse & $\begin{array}{l}\text { No } \\
\text { Yes } \\
\text { Both } \\
\text { Insertive only } \\
\text { Receptive only } \\
\text { Neither }\end{array}$ & $\begin{array}{l}1 \\
1 \cdot 37 \\
1 \\
0 \cdot 46 \\
12 \cdot 37 \\
0.77\end{array}$ & $\begin{array}{l}0 \cdot 40-4 \cdot 68 \\
0 \cdot 12-1 \cdot 76 \\
2 \cdot 78-55 \cdot 02 \\
0 \cdot 21-2 \cdot 80\end{array}$ \\
\hline Partner's status & $\begin{array}{l}\text { Not known HIV+ } \\
\text { Known HIV+ }\end{array}$ & $\begin{array}{l}1 \\
2 \cdot 83\end{array}$ & $1 \cdot 37-5 \cdot 83$ \\
\hline Seen health advisor & $\begin{array}{l}\text { No } \\
\text { Yes }\end{array}$ & $\begin{array}{l}1 \\
0 \cdot 44\end{array}$ & $0 \cdot 18-1 \cdot 08$ \\
\hline Number of clinic attendances & $\begin{array}{l}0 \\
1 \\
2 \text { or more }\end{array}$ & $\begin{array}{l}1 \\
0 \cdot 78 \\
1 \cdot 42\end{array}$ & $\begin{array}{l}0.32-1.88 \\
0.58-3.48\end{array}$ \\
\hline
\end{tabular}

interval and number of tests, there was no systematic difference between values for the cases and the mean of their control(s), Wilcoxon paired signed rank test.

The case notes of both cases and controls were reviewed retrospectively and data collected on the number and type of acute sexually transmitted diseases (STDs) and recorded sexual behaviour during the interval period, defined as the time period between the last two consecutive HIV tests. Data on sexual behaviour included condom use, practice of ano-genital intercourse, and history of sex with a partner or partners known to be HIV antibody positive. The number of sexual partners was not reliably recorded and was therefore not included. Acute STDs included gonorrhoea, first episode of genital herpes, primary and secondary syphilis, chlamydial and nonspecific urethritis. Recurrent herpes simplex and HPV infection were excluded. We also recorded evidence of STDs prior to the last negative test, hepatitis B status, the reasons for repeat testing and contact with a health advisor for pre-test counselling.

\section{Statistical analysis}

Statistical analysis took full account of the matched nature of the data. All the univariate and multivariate odds ratios were calculated using the conditional logistic regression software of the statistical package STATA. To consider the accuracy of the matching, the paired $t$ tests and Wilcoxon paired sign-rank tests were used.

\section{Results}

During the interval period, $27(48 \%)$ of the seroconverters and $25(24 \%)$ of the controls were treated at the clinic for at least one acute STD (table 1). Eleven (20\%) of the seroconverters and $6(6 \%)$ of the controls were treated for at least two and two seroconverters were treated for three, the maximum number of STDs. By univariate analysis presenting with at least one acute STD in the interval period was strongly associated with seroconversion, odds ratio $3 \cdot 5,95 \%$ CI $1 \cdot 6-7 \cdot 6$ (table 2 ).

There were a total of 71 acute STD episodes of which gonorrhoea (27 episodes) and non-specific urethritis (24 episodes) were the most common. The proportions of the various types of STDs were similar in the two groups, for example, $16 / 40(40 \%)$ of the episodes in the seroconverters, and $11 / 31$ $(35 \%)$ in the controls were gonorrhoea (any site).

Before the interval period, almost half of the patients had ever presented with at least one acute STD ( $46 \%$ of cases, $48 \%$ of controls), and this was not associated with seroconversion.

Overall, during the interval period $68 \%$ had reported both receptive and insertive ano-genital intercourse, $13 \%$ insertive only, $11 \%$ receptive only and $8 \%$ neither (table 1 ). By univariate analysis receptive intercourse only was associated with an increased risk of sero- 
Table 3 Condom use by partner's HIV status in cases and controls

\begin{tabular}{lcc}
\hline Condom use & $\begin{array}{l}n(\%) \\
\text { of cases }\end{array}$ & $\begin{array}{l}n(\%) \\
\text { of controls }\end{array}$ \\
\hline All patients & $\mathrm{n}=56$ & $\mathrm{n}=103$ \\
$\quad$ Not at all & $13(23)$ & $5(5)$ \\
Sometimes & $25(45)$ & $25(24)$ \\
Always & $18(32)$ & $73(71)$ \\
With HIV+ partner & $\mathrm{n}=33$ & $\mathrm{n}=19$ \\
Not at all & $5(22)$ & $2(11)$ \\
Sometimes & $10(44)$ & $4(21)$ \\
Always & $8(35)$ & $13(68)$ \\
With partner not known HIV+ & $\mathrm{n}=23$ & $\mathrm{n}=84$ \\
Not at all & $8(24)$ & $3(4)$ \\
Sometimes & $15(46)$ & $21(25)$ \\
Always & $10(30)$ & $21(25)$ \\
\hline
\end{tabular}

conversion relative to those reporting both receptive and insertive intercourse, odds ratio $12 \cdot 4(95 \%$ CI $2 \cdot 8-55 \cdot 0)$ (table 2$)$.

Only $29 \%$ of the seroconverters and $68 \%$ of the controls used condoms consistently (table 3) and lack of condom use was strongly associated with seroconversion. Relative to the men reporting consistent use, the odds ratio of seroconversion amongst those using condoms sometimes was $4.5(95 \%$ CI $1.9-10 \cdot 2)$, and never using condoms was 10.9 (95\% CI $3 \cdot 3-36 \cdot 0$ ) (table 3). For both cases and controls condom use did not differ between those who reported sex with a known HIV antibody positive partner and those who did not.

A history of sex during the interval period with a known HIV antibody positive partner was reported by $41 \%$ of cases and $18 \%$ of controls, and was positively associated with seroconversion, odds ratio $2 \cdot 8(95 \%$ CI $1 \cdot 4-5 \cdot 8)$ (table 2).

Most patients attended the clinic at least once during the interval period $(71 \%$ of cases, $73 \%$ of controls), the median number of attendances was 1 , range $0-8$. Thirty-four percent of seroconverters and $33 \%$ of controls were hepatitis B immune. Whilst a few patients undertook repeat HIV antibody testing because of reported symptoms the major reasons were following an episode of unsafe anogenital intercourse $(20 \%$ of cases, $19 \%$ of controls), sex with an HIV positive partner $(27 \%$ of cases and $15 \%$ of controls), or "other" reasons primarily routine testings (39\% of cases, $55 \%$ of controls). None of these factors was associated with seroconversion.

There was some indication that having seen a health advisor was associated with reduced seroconversion, odds ratio $0.44(95 \%$ CI $0 \cdot 18-1 \cdot 08$ ).

In multivariate analysis adjusted odds ratios (table 4), show that an acute interval STD, inconsistent condom use, and history of sex

Table 4 Multivariate analysis of factors associated with seroconversion

\begin{tabular}{llcl}
\hline Factor & & $\begin{array}{l}\text { Adjusted odds } \\
\text { ratio }\end{array}$ & $\begin{array}{l}95 \% \text { confidence } \\
\text { interval }\end{array}$ \\
\hline STD during interval & None & 1 & - \\
& 1 or more & $4 \cdot 13$ & $1 \cdot 28-13.3$ \\
Known HIV+ partner & No & 1 & - \\
& Yes & $4 \cdot 64$ & $1 \cdot 40-15 \cdot 4$ \\
Condom use & Always & 1 & - \\
& Sometimes & $7 \cdot 90$ & $2 \cdot 16-28 \cdot 9$ \\
& Never & $16 \cdot 2$ & $3 \cdot 04-86.3$ \\
\hline
\end{tabular}

with a known HIV antibody positive partner were all associated with increased seroconversion, after controlling for each other and for age, (included to correct for imprecise matching). Type of ano-genital intercourse practised was not significant, after controlling for the others as this relationship was confounded by condom use. Only $17 \%$ of those reporting just receptive ano-genital intercourse used condoms consistently, as compared to $62 \%$ of those reporting both.

\section{Discussion}

Our study shows that, amongst homosexual men, an acute interval STD, inconsistent or no use of condoms and sexual intercourse with a partner known to be HIV antibody positive are clearly associated with seroconversion. These findings are consistent with both recently reported case control studies and early studies of incident infection. ${ }^{12-15}$ The latter identified receptive ano-genital intercourse, history of an STD (particularly syphilis or gonorrhoea) and a high number of sexual partners to be major factors for transmission. ${ }^{12} 13$

Overall one of the striking features of our results is the relatively high level of unsafe sexual behaviour in the study population. Failure to use condoms regularly was after adjustment for other factors strongly associated with seroconversion. Of concern is that $30 \%$ of the controls also reported inconsistent or no use of condoms and that for both the cases and controls this proportion was similar, irrespective of whether the partner's HIV status was unknown or known to be positive. The reasons why unsafe sexual behaviour occurs are important to determine as, for some men, knowledge of a partner's positive status was clearly insufficient to maintain consistent condom use. Other factors may affect the context in which unsafe sex is practised and include emotional involvement with partners, length and type of relationship, ${ }^{16}$ drug or heavy alcohol use $^{17}$ and current mood. ${ }^{18}$ There is a dearth of knowledge regarding the precise combination of factors that influence unsafe sex, although we cannot ignore the possibility that for some men the practice of unsafe sex will occur irrespective of knowledge, advice or context.

Previous studies have identified sexually transmitted diseases as risk factors for transmission of HIV infection. ${ }^{12-12}$ They are assumed to be a proxy marker of high risk behaviour and may also be a cofactor increasing susceptibility to HIV infection. Our finding that the number and proportion of acute interval STDs was higher amongst the seroconverters implies that STDs continue to be associated with the transmission of HIV infection in this population.

The fact there was an increase in all STDs including non-gonococcal urethritis amongst the seroconverters and that the pattern and type of STDs were similar to the control group suggests that they are a marker of risk behaviour. However, the relative risk of each STD for transmission of HIV may well be very different for different populations. 
Our finding that a life time prior history of an acute STD before the interval period was similarly high in both groups raises the possibility that following their last negative test there was a change in some aspect of either the seroconverters' or controls' sexual behaviour. The reasons and the context in which this has occurred are important to determine as this may have implications for post test counselling. It is encouraging that we identified some association between seeing a health adviser and reduced seroconversion. This may reflect the beneficial effect of counselling as an intervention or could simply mean that the control group are more likely to accept advice to see a health adviser.

Although we are unable to determine accurately from case note review either the total number of recent partners or the frequency of ano-genital intercourse, the study did show that seroconverters were more likely to have had a partner who was known to be HIV antibody positive. This finding supports the suggestion that whilst general adoption of "safer" sex reduces the risk of transmission the frequency of contact with and the number of positive partners may have a strong independent impact on the level of risk. The HIV status of a large proportion of partners in our study was stated to be unknown, thus the true level of exposure for both the cases and the controls to the number of HIV positive partners is uncertain.

Recently reported case control studies have raised the possibility of transmission from orogenital intercourse. ${ }^{14}{ }^{15}$ Our study did not systematically record details of oro-genital sex, but four of the men who seroconverted reported no ano-genital intercourse during the interval period. Although we are unable to confirm this because our data are based on case-note review, it does raise the possibility of transmission of HIV by sexual contact other than ano-genital intercourse. It is important that future studies help to confirm or refute this association with oro-genital sex as this has major implications for health advice to homosexual men.

It is the policy of the clinic to offer routine and voluntary HIV antibody tests to all persons attending, but the results of anonymous unlinked HIV seroprevalence studies suggest that homosexual men who are antibody positive are more likely to be aware of their status than infected heterosexual men and women. ${ }^{7}$ However, knowledge of antibody status is not predictive of sexual behaviour amongst homosexual men, ${ }^{20}$ and our data show that knowledge of partners' HIV status is not associated with safer sex.

There are clearly limitations to a retrospective case note review, not least the uncertainty over the adequacy, verification and completeness of the data, though this is equally true for both the cases and the controls. This most affected our ability to investigate the relationship between seroconversion and the number of sexual partners in the interval period. It is also possible that the incidence of STDs was underestimated as patients may have attended for treatment elsewhere or infections may have been asymptomatic or gone unrecognised. In addition, our findings relate to a selected population attending STD clinics who were willing to undertake repeat HIV antibody tests. The relative importance of the risk factors we identified may be different for unrecognised HIV transmission.

Future studies should try to determine in what context unsafe sexual behaviour is more likely to occur, and the efficacy of interventions to change sexual behaviour. It is clear that gay male attenders at STD clinics, who have repeat antibody tests and who have been exposed to STDs, are a population in whom there is a high risk of seroconversion. This is a population for which targeted prevention programmes are ever more vital.

1 Hart G. AIDS, homosexual men and behavioural change In: Martin CJ and McQueen DV (eds) Readings for a New Public Health 1989 Edinburgh. University Press.

2 Johnson AM, Gill ON. Evidence for recent changes in sexual behaviour in homosexual men in England and Wales. Philostrans $R$, Soc Lond (Biol) 1989;325:153-61.

3 Singaratnam AE, Boag F, Barton SE, Hawkins DA Lawrence DA. Preventing the spread of HIV infection. $B M F$ 1991;302:469.

4 Evans BG, Catchpole MA, Heptonstall J, et al. Sexually transmitted diseases and HIV-1 infection among homosexual men in England and Wales. BMF 1993;306:426-8.

5 Hart G, Dawson J, Fitzpatrick R, et al. Risk behaviour, anti-HIV and anti-HBc prevalence in clinic and nonclinic samples of gay men in England, 1991-1992. AIDS 1993;7:863-9.

6 Miller E, Waight PA, Tedder RS, Sutherland S, Mortime $\mathrm{PP}$, Shafi MS. Incidence of HIV infection in homosexual men in London 1988-94. BMF 1995:311:545.

7 Public Health Laboratory Service and Collaborators. Unlinked anonymous HIV prevalence monitoring proUnlinked anonymous HIV prevalence monitoring programme England and

8 Communicable Disease Report (1993) The incidence and prevalence of AIDS and other severe HIV disease in England and Wales for 1992-1997: projections using data to the end of fune 1992. Report of a working group (Chairman: Pro NE Day); 3 (Supp 1):S1-S17.

9 Winkelstein W, Lyman DM, Padian N, et al. Sexual practice and risk of infection with the immune immunodeficiency virus. $\mathcal{F} A M A$ 1987;257:321-5.

10 Chmiel JS, Detels R, Kaslow RA, Van Raden M, Kingsley LA, Brookmuyer $R$. Factors associated with prevalent human immunodeficiency virus (HIV) infection in a multicenter AIDS cohort study. Am $¥$ Epidemiol 1987; 126:568-77.

11 Evans BA, Maclean KA, Dawson SG, et al. Trends in sexua behaviour and risk factors for HIV infection among homosexual men 1984-1987. BMf 1989;298:215-8.

12 Darrow WW, Echenberg DF, Jaffe HW, et al. Risk factor for Human immunodeficiency virus (HIV) infections in homosexual men. Am $\mathcal{F}$ Public Health 1987;77:479-83.

13 Kingsley LA, Detels R, Kaslow R. Risk factors for seroconversion to Human immunodeficiency virus among male homosexuals. Results from the multicenter AIDS cohor study. Lancet 1987;1:345-8.

14 Samuel M, Hessol N, Shiboski S, Angle R, Speed T, Winkelstein $\mathrm{W}$. Factors associated with Human immunodeficiency virus seroconversion in homosexual men in 3 San Francisco cohort studies 1984-1989. AIDS 1993;6: 303-12.

15 Kett JPM, Albrecht van Lent N, Sandfort TGM, Coutinho RA, Van Griensven GHP. Oro-genital sex and the transmission of HIV among homosexual men. AIDS 1992;6: 223-6.

16 McLean J, Boulton M, Brookes M, et al. Regular partner and risky behaviour: why do gay men have unprotected intercourse? AIDS Care 1994;6:333-44.

17 Pnkower L, Dew MA, Kingsley L, et al. Behavioural health and psychosocial factors and risk for HIV infection among sexually active homosexual men. Multicentre AIDS cohort study. Am 7 Public Health 1991;81:194-8.

18 Gold $R$ and Skinner $M$. Situational factors and thought processes associated with unprotected intercourse in young gay men. AIDS 1992;6:1021-30.

19 Kuiaen CL, Van Griensven GJ, de Vroome EM, Coutinho RA. Risk fan Griensven GJ, de Vroome EM, Coutinh RA. Risk factors and changes in sexual behaviour in male homosexuals who seroconverted for Human immuno523-30.

20 Dawson J, Fitzpatrick R, Reeves G, et al. Awareness of sexual partners' HIV status as an influence upon high risk sexual behaviour among gay men. AIDS 1994;8:837-41. 